\title{
Calculating Masses of Pentaquarks Composed of Baryons and Mesons
}

\author{
M. Monemzadeh, N. Tazimi, and Sh. Babaghodrat \\ Department of Physics, University of Kashan, Ghotb Ravandi Boulevard, Kashan 87317-51167, Iran \\ Correspondence should be addressed to N. Tazimi; nt_physics@yahoo.com
}

Received 24 December 2015; Accepted 10 May 2016

Academic Editor: Antonio Ereditato

Copyright ( 2016 M. Monemzadeh et al. This is an open access article distributed under the Creative Commons Attribution License, which permits unrestricted use, distribution, and reproduction in any medium, provided the original work is properly cited. The publication of this article was funded by $\mathrm{SCOAP}^{3}$.

\begin{abstract}
We consider an exotic baryon (pentaquark) as a bound state of two-body systems composed of a baryon (nucleon) and a meson. We used a baryon-meson picture to reduce a complicated five-body problem to simple two-body problems. The homogeneous Lippmann-Schwinger integral equation is solved in configuration space by using one-pion exchange potential. We calculate the masses of pentaquarks $\theta_{c}(u u d d \bar{c})$ and $\theta_{b}(u u d d \bar{b})$.
\end{abstract}

\section{Introduction}

There are two types of hadrons, baryons and mesons. Baryons are equivalent to the bound states of three quarks, and mesons are known to be the bound states of a quark and an antiquark. However, QCD describes mesons and baryons even with a more intricate structure. There are anomalous mesons such as $g 9 g, q \bar{q} g$, and $q \bar{q} q \bar{q}$, as well as exotic baryons like $q 9 q 9$, $9999 \bar{q}$, and so forth. Pentaquarks are baryons with at least four quarks and one antiquark. In exotic pentaquarks, the antiquark has a flavor different from the other four quarks.

Exotic hadrons containing at least three valence quarks are being studied fairly extensively in modern physics. Although there are hundreds of ordinary hadrons, exotic ones have not been found stable yet. However, QCD does not reject their existence. Pentaquark $\theta^{+}$, studied in photo production experiments $[1,2]$, is a prototype of exotic hadrons in light and strong quark sector. Theoretically, hadronic reactions contribute to $\theta^{+}$production more vividly than other types of reactions.

The quark model is commonly used to describe hadrons. In this model, mesons are described as $q \bar{q}$ and baryons as three-quark composite particles. In a more microscopic view, QCD usually serves to describe the strong interaction. According to Lipkin [3, 4] and Gignoux et al. [5], among pentaquarks, the five-quark anticharmed baryons of
$P^{0}=[u u d \bar{c} s]$ and $\bar{p}=[u d d \bar{c} s]$ or similar antibeauty baryons are the most bound.

A lot of experimental evidence on the existence of exotic hadrons has been found since 2003. Exotic hadrons' quantum numbers cannot be justified based on two- and threequark bound states. Pentaquarks of $9999 \bar{q}$ form are examples of exotic baryon states. Conjugation quantity of $C$ charge is not an accurate quantum number for baryons, and all combinations of total spin $J$ and parity $P$ can exist. However, an exotic baryon combination can be readily identified by its electric charge $Q$ and its strangeness $S$. Some evidence has been reported during the last few years. For example, the pentaquark $\theta^{++}$was proven to exist in Hermes experiment in Hamburg, Germany [6, 7].

For exotic baryons, we consider the following:

$\theta^{+}$: the existence of this exotic baryon was predicted in chiral solution model [8]. It has $S=+1, J^{P}=1 / 2^{+}$, and $I=0$. It is a narrow light-mass particle of $1540 \mathrm{MeV}$. These attributes initially made $\theta^{+}$a subject of experimental observation by LEPS [9]. The most suitable hadronic decay mode to identify it is $\theta^{+} \rightarrow$ $K^{0} p$.

$\theta_{c}$ and $\theta_{b}$ : the existence of the bound exotic hadron $\theta_{c}$ was predicted through bound Skyrmion approach. This particle has a mass of $2650 \mathrm{MeV}$ and quantum 
numbers $J^{P}=1 / 2^{+}$and $I=0$. An experiment [10] showed a positive signal at a mass of about $3.1 \mathrm{GeV}$, but it was not confirmed later [11]. In strongly bound states, the decay mode $K^{+} \pi^{-} \pi^{-} p$ is easy to identify. Likewise, the mass of $\theta_{b}$ with the same quantum numbers $J^{P}=1 / 2^{+}$and $I=0$ was predicted to be $5207 \mathrm{MeV}$. The possible weak decay mode is $K^{+} \pi^{-} \pi^{-}+$ $\pi^{+}+p$.

$\theta_{c s}$ : it is the five-quark state with $J^{P}=1 / 2^{-}$and $I=0$. In a quark model which includes color-spin interaction, it can be bound and despite its strong decay, it becomes stable [12]. The mass dependent on the model parameters is predicted to be $2420 \mathrm{MeV}$. This state was traced in the Fermilab E791 experiment via $\phi \pi p$ mode [13] and $K^{* 0} K^{-} p$ mode [14].

Lippmann-Schwinger equation for two-body bound states is solved in Section 2. In Section 3, the procedure of the study is given and pentaquark masses are calculated.

\section{Lippmann-Schwinger Equation for Two-Body Bound States}

In this part, the binding energy of the entire system (pentaquark) is calculated by numerical solution of homogeneous Lippmann-Schwinger equation for each subsystem of bound meson and baryon. Schrodinger equation for a two-body bound state with the potential $V$ runs as the following integral equation $[16,17]$ :

$$
\begin{aligned}
& \psi_{b}(r)=-\frac{m}{4 \pi} \int_{0}^{\infty} d r^{\prime} r^{\prime 2} \int_{-1}^{1} d x^{\prime} \\
& \cdot \int_{0}^{2 \pi} d \phi^{\prime} \frac{\exp \left(-\sqrt{m\left|E_{b}\right|}\left|r-r^{\prime}\right|\right)}{\left|r-r^{\prime}\right|} V\left(r^{\prime}\right) \psi_{b}\left(r^{\prime}\right),
\end{aligned}
$$

where $E_{b}$ stands for the binding energy of the two-body bound system (meson + baryon). When the interaction potential is considered as $r$ function, (1) is of the following eigenvalue form

$$
K\left(E_{b}\right)\left|\psi_{b}\right\rangle=\lambda\left(E_{b}\right)\left|\psi_{b}\right\rangle
$$

$\lambda=1$ is the highest positive eigenvalue. The eigenvalue equation is solved through direct method [16-19] using a regularization method $[20,21]$. To discretize the integrals, Gauss-Legendre method [22] is employed. 200 grid points are considered for $r$ and $r^{\prime}$, whereas 100 mesh points are quite enough for $x^{\prime}$. The kernel of integral equation is diagonized to find $\lambda=1$ in the eigenvalue spectrum. The energy corresponding to $\lambda=1$ will be the system's binding energy.

\section{Numerical Results and Discussion}

We consider a pentaquark as a bound state of a two-particle system formed by a baryon and a meson. The hadronic molecular structure consisting of a baryon and a meson is shown in Figure 1.

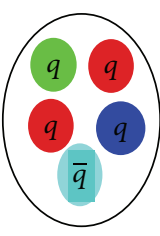

Pentaquark

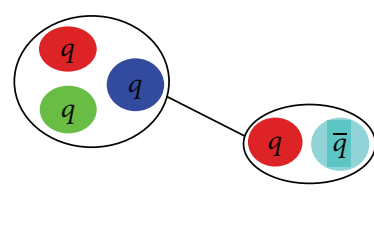

Hadronic molecule

FIgure 1: Pentaquark.

Interaction potential has an essential role in solving the eigenvalue equation (5). Different potentials have been introduced for meson-baryon interaction. Yukawa potential (screened Coulomb potential) is proposed as one of the appropriate ones $[15,23]$. This study deals with exotic baryon states created by a meson and a nucleon. The $\pi$ exchange potential is among the most prominent meson exchange forces. $\pi$ is the lightest hadron that can be exchanged between a meson and a nucleon. Therefore, we consider only $\pi$ exchange, and $\rho$ and $\omega$ meson exchange will be elaborated on in our subsequent works. One-pion exchange potential (OPEP) is of the following form [15]:

$$
\begin{aligned}
& V_{\pi}(r) \\
& \quad= \begin{cases}\left(\vec{I}_{N} \cdot \vec{I}_{H}\right)\left(2 S_{12} V_{T}(r)+4 \vec{S}_{N} \cdot \vec{S}_{l}\right) V_{c}(r), & r>r_{0}, \\
V_{0}, & r<r_{0},\end{cases}
\end{aligned}
$$

where for $r>r_{0}$

$$
\begin{aligned}
& V_{\pi}(r)=\left(\vec{I}_{N} \cdot \vec{I}_{H}\right)\left(2 S_{12} V_{T}(r)+4 \vec{S}_{N} \cdot \vec{S}_{l}\right) V_{c}(r) \\
& \quad=\left(I^{2}-I_{N}^{2}-I_{H}^{2}\right)\left(2 S_{12} V_{T}(r)+K^{2}-S_{N}^{2}-S_{l}^{2}\right) V_{c}(r),
\end{aligned}
$$

where

$$
\begin{aligned}
V_{c}(r) & =\frac{g_{H} g_{A}}{2 \pi f_{\pi}^{2}}\left(m_{\pi}^{2}\right) \frac{e^{-m_{\pi} r}}{3 r}, \\
V_{T}(r) & =\frac{g_{H} g_{A}}{2 \pi f_{\pi}^{2}}\left(m_{\pi}^{2}\right) \frac{e^{-m r}}{6 r}\left(\frac{3}{r^{2}}+\frac{3}{r}+1\right), \\
V_{0} & =-62.79(\mathrm{MeV}) \text { or }-276(\mathrm{MeV}),
\end{aligned}
$$

where $g_{A}, f_{\pi}, m_{\pi}$, and $g_{H}$ are axial coupling constant, pion decay constant, pion mass, and heavy-meson coupling constant, respectively. $I$ is the total isospin of meson-nucleon system and

$$
S_{12} \equiv 4\left[3\left(\vec{S}_{N} \cdot \widehat{r}\right)\left(\vec{S}_{l} \cdot \widehat{r}\right)-\vec{S}_{N} \cdot\left(\vec{S}_{l}\right)\right]
$$

Inserting $I_{N}$ (nucleon isospin), $I_{H}$ (meson isospin), $S_{N}$ (nucleon spin), $S_{l}$ (the lightest quark's spin in the meson), and $K=S_{N}+S_{l}$ into the potential, we presented hadronic molecular structure of two pentaquarks in Table 2.

In this calculation we have ignored the tensor term $S_{12}$ for studying the pentaquarks ground state [24]; hence, we used the central part of Yukawa potential. Pentaquark 
TABLE 1: Potential parameters used for the bound state of heavy pentaquarks [15].

\begin{tabular}{lc}
\hline Parameter & Value \\
\hline$g_{A}$ & 1.27 \\
$f_{\pi}$ & $131 \mathrm{MeV}$ \\
$g_{H}$ & -0.59 \\
$m_{\pi}$ & $138 \mathrm{MeV}$ \\
$m_{N i}$ & $938.92 \mathrm{MeV}$ \\
$m_{B}$ & $5279 \mathrm{MeV}$ \\
$m_{D}$ & $1867 \mathrm{MeV}$ \\
\hline
\end{tabular}

TABLE 2: Hadronic molecular structure of pentaquarks.

\begin{tabular}{lc}
\hline Hadronic structure & Pentaquark (meson + nucleon) \\
\hline$\theta_{c}(u u d d \bar{c})$ & $u u d / u d d, d \bar{c} / u \bar{c}$ \\
$\theta_{b}(u u d d \bar{b})$ & $u u d / u d d, d \bar{b} / u \bar{b}$ \\
\hline
\end{tabular}

binding energy is defined as the energy used when breaking a pentaquark into its components, that is, meson and baryon. We adopted the following constants for the bound state of heavy pentaquarks from [15] (Table 1).

Pentaquark masses are calculated according to

$$
M(\text { pentaquark })=m_{\text {meson }}+m_{\text {baryon }}+E_{b} .
$$

In order to find the binding energy $\left(E_{b}\right)$, first we solve Lippmann-Schwinger equation for the two-body system. In this approach, the kernel is diagnosed and the eigenvalue spectrum is identified (spin-spin interaction in the potential and spin splitting are ignored). The data required include reduced mass of mesons and nucleons, proposed binding energy, potential coefficients, $r_{0}=1$ or $1.5 \mathrm{fm}$, and $r$-cutoff $=$ $20 \mathrm{fm}$. Table 3 shows the binding energies that we found.

We adopted Yukawa potential from [15] and our calculated energies are in good agreement with [15]. We present pentaquark masses in Table 4.

\section{Results and Discussion}

In this paper, we solved Lippmann-Schwinger equation for pentaquark systems. We managed to obtain the binding energy and used it to calculate the masses of these systems. The pentaquark is considered as the bound state of a baryon and a heavy meson. We used a baryon-meson picture to reduce a complicated five-body problem to one simple twobody problem. In Table 4, we have listed our numerical results for masses of pentaquark systems, and pentaquark masses are compared with the results obtained in [25-27].

Our method is appropriate for investigating tetraquark systems too. In our previous work, we investigated the tetraquark as the bound state of a heavy-light diquark and antidiquark. We used the diquark-antidiquark picture to reduce a complicated four-body problem to simple two-body problems.

We analyzed diquark-antidiquark in the framework of a two-body (pseudopoint) problem. We made use of the potential coefficients proposed by Ebert et al. We solved
TABLE 3: The calculated binding energies $(\mathrm{MeV})$ for $\theta_{c}$ ( $D$ meson) and $\theta_{b}$ ( $B$ meson) for $I=0$. Column A: $V_{0}=-276 \mathrm{MeV}$ and $r_{0}=$ $1 \mathrm{fm}$; column B: $V_{0}=-62.79 \mathrm{MeV}$ and $r_{0}=1.5 \mathrm{fm}$.

\begin{tabular}{lcccc}
\hline Pentaquark & $\mathrm{A}$ & $\mathrm{B}$ & $\mathrm{A}[15]$ & $\mathrm{B}[15]$ \\
\hline$\theta_{c}$ & 113.32 & 8.21 & 113.99 & 8.45 \\
$\theta_{b}$ & 140 & 15.1 & 139.48 & 15.46 \\
\hline
\end{tabular}

TABLE 4: Pentaquark calculated masses $(\mathrm{MeV})$ for $I=0$.

\begin{tabular}{lccc}
\hline Pentaquark & Mass $(\mathrm{A})$ & Mass $(\mathrm{B})$ & Mass in [25-27] \\
\hline$\theta_{c}$ & 2665.92 & 2790.82 & 2650 \\
$\theta_{b}$ & 6104.6 & 6209.71 & 6391 \\
\hline
\end{tabular}

Lippmann-Schwinger equation numerically for charm diquark-antidiquark systems and found the eigenvalues to calculate the binding energies and masses of heavy tetraquarks with hidden charms [28].

\section{Competing Interests}

The authors declare that there is no conflict of interests regarding the publication of this paper.

\section{Acknowledgments}

This work is supported by University of Kashan under Grant no. 65500.6. The authors also express their greatest gratitude to Dr. M. R. Hadizadeh for his helpful technical comments on the numerical solution of the LS integral equation and also for comments that helped them to revise the text of the paper.

\section{References}

[1] T. Nakano, D. S. Ahn, J. K. Ahn et al., "Evidence for a narrow $S=$ +1 baryon resonance in photoproduction from the neutron," Physical Review Letters, vol. 91, no. 1, Article ID 012002, 2003.

[2] T. Nakano, N. Muramatsu, D. S. Ahn et al., "Evidence for the $\Theta^{+}$in the $\gamma d \rightarrow K^{+} K^{-} p n$ reaction by detecting $K^{+} K^{-}$pairs," Physical Review C, vol. 79, Article ID 025210, 2009.

[3] H. J. Lipkin, "New possibilities for exotic hadrons: anticharmed strange baryons," Physics Letters B, vol. 195, no. 3, pp. 484-488, 1987.

[4] H. J. Lipkin, "Multiquark physics-the great challenge for future directions in the interplays between particle and nuclear physics," Nuclear Physics A, vol. 478, pp. 307-324, 1988.

[5] C. Gignoux, B. Silvestre-Brac, and J. M. Richard, "Possibility of stable multiquark baryons," Physics Letters B, vol. 193, no. 2-3, pp. 323-326, 1987.

[6] A. Airapetianae, N. Akopovae, and Z. Akopov, "Evidence for a narrow $|S|=1$ baryon state at a mass of $1528 \mathrm{MeV}$ in quasi-real photoproduction," Physics Letters B, vol. 585, no. 3-4, pp. 213222, 2004.

[7] A. Airapetian, N. Akopov, Z. Akopov et al., "Search for an exotic $S=-2, Q=-2$ baryon resonance at a mass near $1862 \mathrm{MeV}$ in quasireal photoproduction," Physical Review D, vol. 71, no. 3, Article ID 032004, 5 pages, 2005.

[8] T. Nakano, D. S. Ahn, J. K. Ahn et al., "Evidence for a narrow $S=$ +1 baryon resonance in photoproduction from the neutron," Physical Review Letters, vol. 91, Article ID 012002, 2003. 
[9] D. Diakonov, V. Petrov, and M. Polyakov, "Exotic anti-decuplet of baryons: prediction from chiral solitons," Zeitschrift für Physik A: Hadrons and Nuclei, vol. 359, no. 3, pp. 305-314, 1997.

[10] A. Aktas, V. Andreevz, T. Anthonis et al., "Evidence for a narrow anti-charmed baryon state," Physics Letters B, vol. 588, no. 1-2, pp. 17-28, 2004.

[11] J. M. Link, P. M. Yagera, J. C. Anjos et al., "Search for a strongly decaying neutral charmed pentaquark," Physics Letters B, vol. 622, no. 3-4, pp. 229-238, 2005.

[12] H. J. Lipkin, "New possibilities for exotic hadronsanticharmed strange baryons," Physics Letters B, vol. 195, no. 3, pp. 484-488, 1987.

[13] E. M. Aitala, S. Amato, J. C. Anjos et al., "Search for the Pentaquark via the $P_{\overline{c s}}^{0} \rightarrow \varphi \pi p$ decay," Physical Review Letters, vol. 81, pp. 44-48, 1998.

[14] E. M. Aitala, S. Amato, J. C. Anjos et al., "Search for the pentaquark via the $P_{\overline{c s}}^{0} \rightarrow K^{* 0} K^{-} p$ decay," Physics Letters $B$, vol. 448, no. 3-4, pp. 303-310, 1999.

[15] T. D. Cohen, P. M. Hohler, and R. F. Lebed, "On the existence of heavy pentaquarks: The large $N_{c}$ and heavy quark limits and beyond," Physical Review D, vol. 72, no. 7, Article ID 074010, 2005.

[16] M. Monemzadeh, M. Hadizadeh, and N. Tazimi, "Identification of the mass and stability interval of strong potential in heavy mesons," International Journal of Theoretical Physics, vol. 50, no. 3, pp. 737-743, 2011.

[17] M. R. Hadizadeh, "Comment on 'Tetraquarks as diquarkantidiquark bound systems," The European Physical Journal C, vol. 75, article 281, 2015.

[18] N. Tazimi, M. Monemzadeh, and M. R. Hadizadeh, "Description of heavy quark ms mass by lippmann-schwinger equation," International Journal of Theoretical Physics, vol. 51, no. 9, pp. 2871-2877, 2012.

[19] N. Tazimi, M. Monemzadeh, and M. R. Hadizadeh, "Heavy mesons spectroscopy," International Journal of Theoretical Physics, vol. 52, no. 7, pp. 2329-2334, 2013.

[20] M. R. Hadizadeh and A. Khaledi-Nasab, "Heavy tetraquarks in the diquark-antidiquark picture," Physics Letters, Section B: Nuclear, Elementary Particle and High-Energy Physics, vol. 753, pp. 8-12, 2016.

[21] M. R. Hadizadeh and L. Tomio, "Solution of two-body bound state problems with confining potentials," in Proceedings of the XI Hadron Physics, vol. 1296 of AIP Conference Proceedings, p. 334, São Paulo, Brazil, March 2010.

[22] S. Nakamura, Applied Numerical Methods with Software, Prentice Hall, 1998.

[23] Y. Yamaguchi, S. Ohkoda, S. Yasui, and A. Hosaka, "Exotic baryons from a heavy meson and a nucleon: negative parity states," Physical Review D-Particles, Fields, Gravitation and Cosmology, vol. 84, no. 1, Article ID 014032, 2011.

[24] D. Ebert, R. N. Faustov, and V. O. Galkin, "Quark-antiquark potential with retardation and radiative contributions and the heavy quarkonium mass spectra," Physical Review D, vol. 62, Article ID 034014, 2000.

[25] S. Cho, T. Furumoto, T. Hyodo et al., "Identifying multiquark hadrons from heavy ion collisions," Physical Review Letters, vol. 106, no. 21, Article ID 212001, 2011.

[26] D. Diakonov, "Prediction of new charmed and bottom exotic pentaquarks," https://arxiv.org/abs/1003.2157.

[27] M. Karliner, Pentaquark Workshop, Jlab, November 2003.
[28] M. Monemzadeh, N. Tazimi, and P. Sadeghi, "Tetraquarks as diquark-antidiquark bound systems," Physics Letters, Section B: Nuclear, Elementary Particle and High-Energy Physics, vol. 741, pp. 124-127, 2015. 

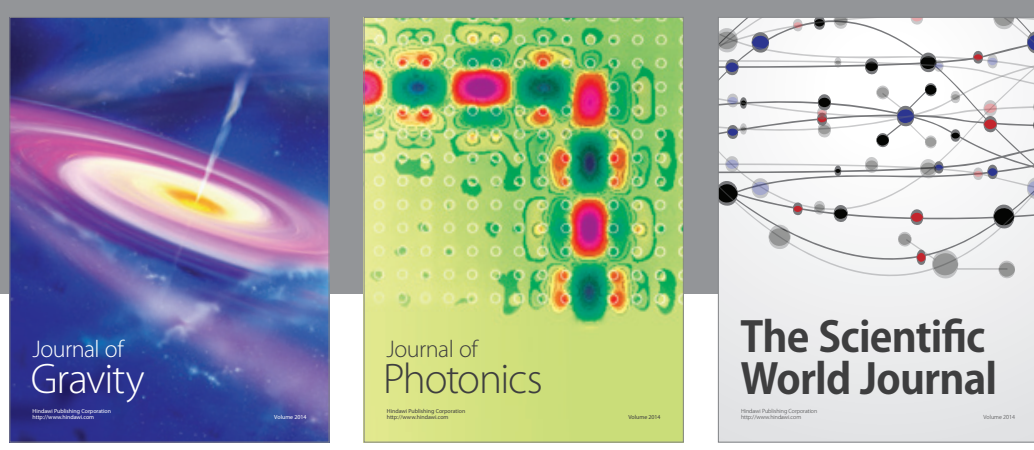

The Scientific World Journal
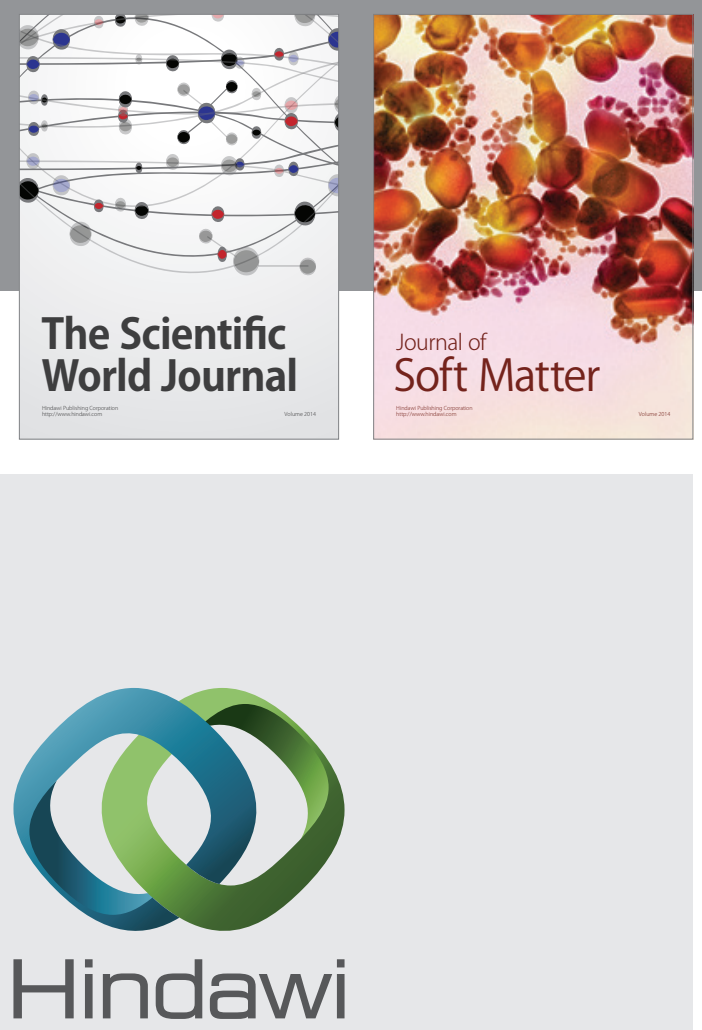

Submit your manuscripts at

http://www.hindawi.com

nternational Journal of

Statistical Mechanics
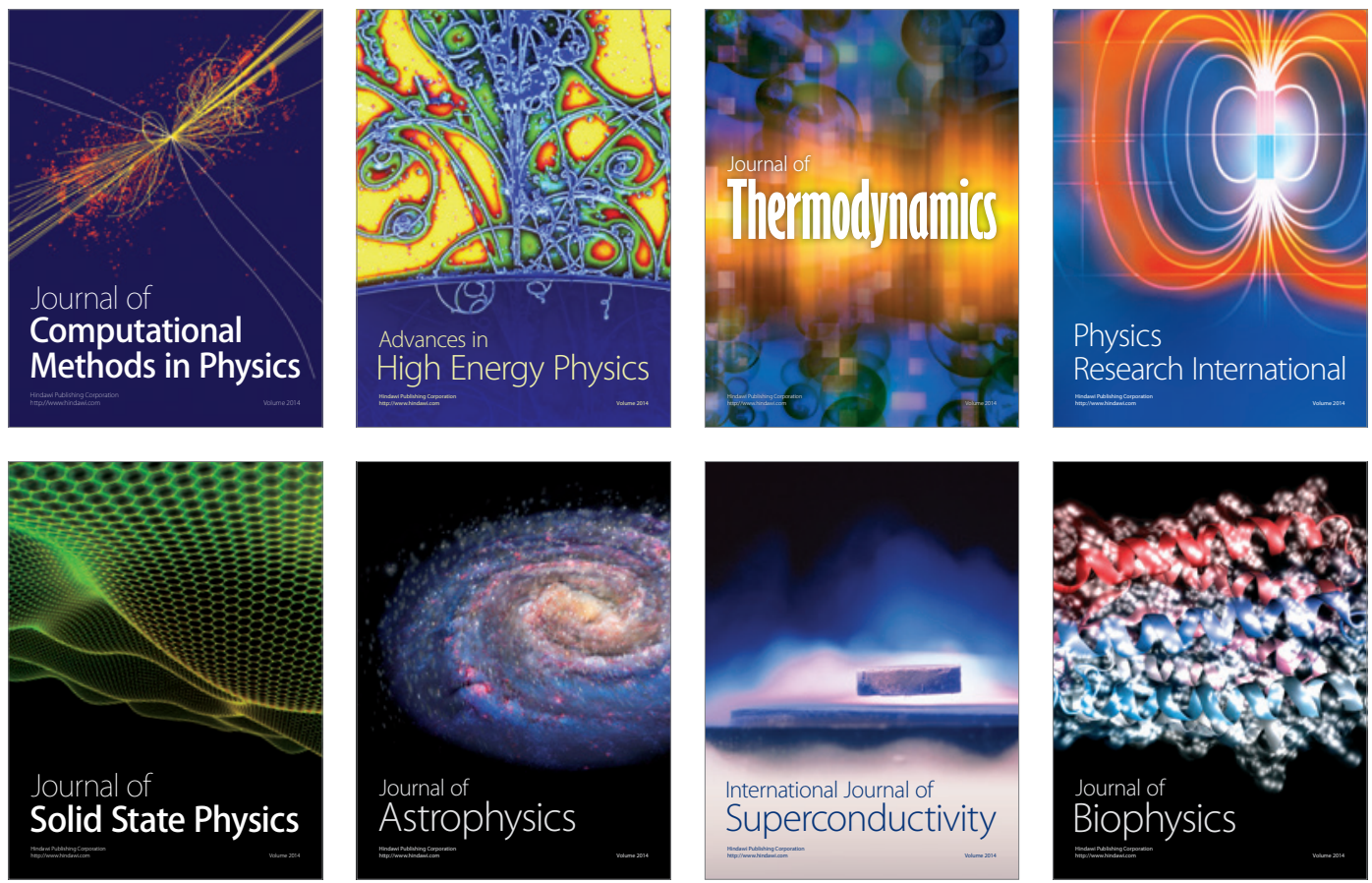
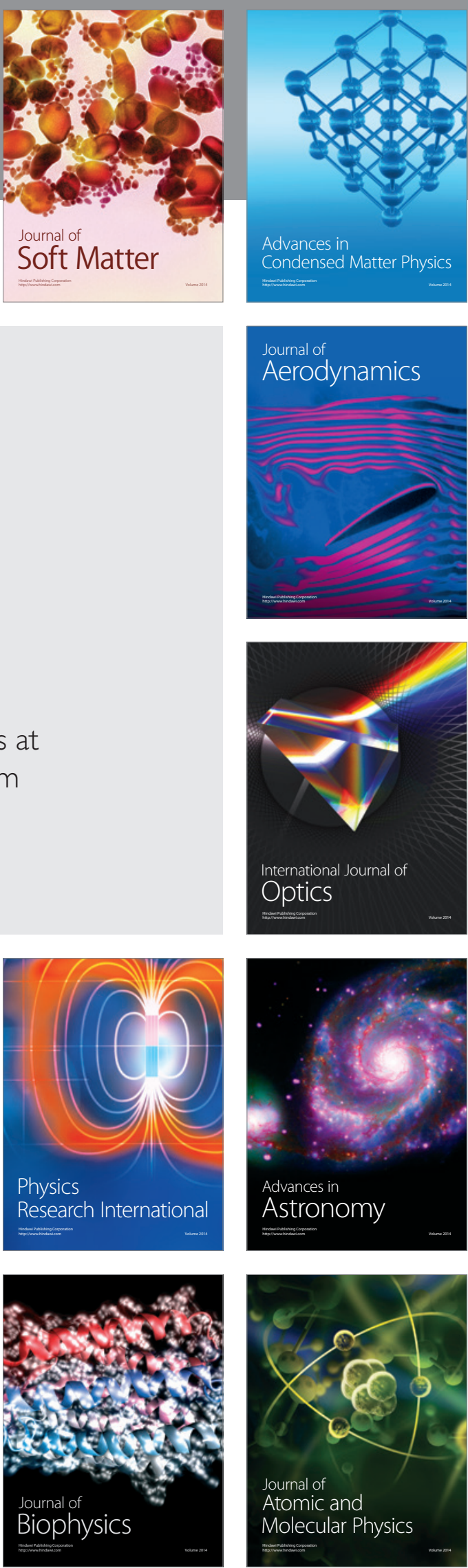